\title{
An EP-SAW for Measurements of Particulate Matter in Ambient Air
}

S.M.Stanley ${ }^{*}$, G.McHale $^{*}$, M.I.Newton ${ }^{*+}$, C.J.Percival ${ }^{\&}$ and C.R.Evans*

* School of Biomedical and Natural Sciences, Nottingham Trent University, Clifton Lane, Nottingham UK NG11 8NS

\& School of Earth, Atmospheric and Environmental Sciences, Sackville Building, The University of Manchester, Sackville Street, P.O. Box 88, Manchester, M60 1QD UK

+ corresponding author, e-mail michael.newton@ntu.ac.uk

telephone +44 115 8483365, fax +44 1158486636

\begin{abstract}
In this article we demonstrate the feasibility of combining the two proven technologies of electrostatic precipitators (EP) and surface acoustic wave devices (SAW) for real time applications in particulate monitoring. A plastic cyclone (Higgins-Dewell), used for personal respirable dust sampling, has been modified to include an ionizing source and a Rayleigh SAW device with biased collection plate in place of the usual filter. The flow rate of the air through the cyclone allows size selectivity of particulate matter and we present data showing the effect of the collection plate bias of the collection efficiency.
\end{abstract}




\section{Introduction}

In this article we present preliminary data to demonstrate the feasibility of combining the two proven technologies of electrostatic precipitators (EP) and surface acoustic wave devices (SAW) for real time application in particulate monitoring. Electrostatic precipitation of particles has been routinely used in applications, such as the collection of airborne microorganisms [1], sampling of cloud and fog droplets [2] and the determination of lead in atmospheric aerosols [3]. Electrostatic precipitators operate on the principle of the attraction of charged particles to an oppositely charged collector. A voltage is applied to a collector plate and the charged particulates then deposit on the plate. Electrostatic precipitators have been reported with high collection efficiencies for particulate matter (up to 99\%) over a wide range of sizes (0.05-5 $\mu \mathrm{m})$.

A surface acoustic wave (SAW) is a combined mechanical and electromagnetic field disturbance that is localised to around one wavelength of the surface of a solid [4]. On a piezoelectric substrate the wave can be generated by applying a radio frequency voltage to a metal interdigital transducer (IDT). The spacing of the fingers of the IDT determines the wavelength and this together with the substrate orientation, which defines the propagation speed, determines the frequency of operation. A SAW device using a delay line configuration provides a free surface area between two IDT that can be used as a sensor. As the wave is surface localised, any mass deposited on the propagation path changes the speed of propagation. Surface acoustic wave devices are highly surface mass sensitive but are not intrinsically selective however they do have the potential to provide real-time measurement; the use of a SAW device for aerosol 
monitoring was demonstrated in 1991 by Bowers et. al. [5] using olive oil as the test aerosol. In a previous report EP have been used in conjunction with quartz crystal microbalances (QCM), an acoustic wave device with a lower sensitivity than SAW devices, in a prototype personal dust monitor [6]. In this article we demonstrate the feasibility of combining SAW and EP technologies for real time particulate monitoring.

\section{Experimental}

The SAW devices used were dual IDT delay lines fabricated in house on $128^{\circ}$ rotated Y-cut lithium niobate and operating at a fundamental frequency of $86 \mathrm{MHz}$; on this cut a Rayleigh wave will propagate. The IDT consisted of sputtered Ti/Au of thickness $0.10 \mu \mathrm{m}$ and utilised a split finger design to minimise the triple transit interference. Each IDT was of length $40 \lambda_{\text {IDT }}$ and aperture $65 \lambda_{\text {IDT }}$, where the wavelength $\lambda_{\text {IDT }}=45$ $\mu \mathrm{m}$. Fingers widths were $6.75 \mu \mathrm{m}$ and spacing $4.5 \mu \mathrm{m}$ with an IDT separation of 7 mm. A $6 \mathrm{~mm}$ metal pad of $\mathrm{Ti} / \mathrm{Au}$ was deposited between the IDT to provide a collection plate onto which a bias voltage could be applied. The SAW devices were mounted in a modified (Higgins-Dewell) cyclone as shown in Figure 1. Cyclones are routinely used for personal dust monitors as they size select on the basis of the flow rate of the air drawn through them; the usual flow rate for personal monitoring of 2.2 1/min gives a D50 (50\% of particles passed) at $5 \mu \mathrm{m}$. The ionising radiation source from a domestic smoke detector (Americium) was situated directly in the path of the gas flow in the upper chamber of the cyclone; the larger particles have been discarded into the grit pot by this stage in the device. The SAW was mounted behind the Americium source as this arrangement prevented both gravity-induced settlement on the SAW and direct impaction from the air stream; only when a bias voltage was 
applied to the SAW was particulate matter attracted to the SAW. The SAW parameters were monitored using an Agilent 8712ET network analyser making four measurements per second. Any mass deposition on the device surface resulted in a phase change measured at the network analyser. The test material was produced using a condensation aerosol generator (TOPAS SLG 270) to provide a mono-dispersed aerosol. The aerosol generation principle is based on controlled heterogeneous condensation. Vaporized aerosol matter, Di-2-ethyl hexyl sebacate (DEHS), was recondensed onto small sodium chloride particles serving as condensation nuclei. Particle size and number concentration were varied by the operating parameters and this was monitored using the TOPAS PAM510/s monitor. The cyclone was attached to a personal monitoring pump equipped with a flow rate monitor and the inlet to the cyclone situated such that it could draw off a small fraction of the generated particulates; the collection efficiency was estimated using standard filters situated between the outlet of the cyclone and the pump.

\section{Results and Discussion}

Using our experimental arrangement, the greatest stability of particle size and concentration was obtained at high concentrations $\left(>10^{5}\right.$ per $\left.\mathrm{cm}^{3}\right)$. Extended periods of exposure at these concentrations rapidly saturated the SAW device as they are orders of magnitude greater than would be encountered in routine environmental monitoring; as such only a small volume was drawn off the main gas stream through the cyclone. A stable aerosol of size $2.7 \pm 0.2 \mu \mathrm{m}$ was established and the SAW device allowed to reach a steady state with the sampling pump running before a collection plate bias was applied. In figure 2 we show the effect of applied collection plate bias on the SAW phase with the bias increasing in steps of $100 \mathrm{~V}$ every 100 
seconds; the rate of phase change from this data is shown in figure 3. From this data we can see that the collection efficiency increases with applied voltage however a maximum collection rate has not have been achieved over the range of voltages that were applied; we would expect a plateau region where maximum collection was achieved and an increase in bias would not affect the collection efficiency. The maximum voltage in the data presented was limited by problems associated with the separation between the SAW IDT and the bias plate; a modified design should allow voltages of several $\mathrm{kV}$ to be applied if required.

A number of other technical issues remain to be resolved before such a device can be considered as a field instrument. The use of standard filters has given the collection efficiency with the applied bias of $900 \mathrm{~V}$ compared to the unbiased device of around 30\%. This low collection efficiency is in part due to the ionising source producing both positively and negatively charged particles. This is clearly demonstrated by reversing the polarity of the applied bias where a near identical collection efficiency with applied bias is observed. The use of two identical SAW devices, one with a positive bias and one with a negative bias, would allow the collection of both types of charged particulate giving a collection efficiency in excess of $60 \%$; the availability of small and inexpensive dc to dc converters providing several $\mathrm{kV}$ from typically a $12 \mathrm{~V}$ supply makes this a viable solution even for field based instruments. The collection plate bias has also not reached a plateau region at the highest applied bias due to SAW device collection plate design considerations. A modified SAW device operating in a voltage plateau region would further increase this collection efficiency. For any field instrument the SAW devices need to be incorporated into resonator circuits so that only changes in the resonant frequency 
need be measured; the use of a third SAW device without a collection plate could also be incorporated to allow for ambient changes such as temperature drift.

\section{Conclusion}

In this article we have presented data showing that a particulate aerosol may be charged using an ionizing radiation source and detected using the metalised path of a SAW device built into a standard cyclone. The collection efficiency of the device increases with the applied collection plate bias voltage up $900 \mathrm{~V}$. This work has demonstrated the feasibility of combining the two proven technologies of electrostatic precipitators (EP) and surface acoustic wave devices (SAW) for real time application in particulate monitoring.

\section{Acknowledgements}

The authors acknowledge EPSRC for funding under research grant GR/R36718/01.

\section{References}

[1] G. Mainelis, S.A. Grinshpun, K. Willeke, T. Reponen, V. Ulevicius, and P.J. Hintz, Aerosol Sci. Tech. 30, 127 (1999).

[2] B. Tenberken and K. Bachmann, Atmos. Environ. 32, 1757 (1998)

[3] G. Torsi, P. Reschiglian, M.T. Lippolis and A. Toschi, Microchemical Journal 53, 437 (1996)

[4] A.A. Oliner, Acoustic Surface Waves, Springer-Verlag. New York. (1977)

[5] W.D. Bowers, R.L. Chuan and T.M. Duong, 62, 1624 (1991)

[6] L.W. Wilson, M.J. Hepher, D. Reilly and J.D.C. Jones, Meas. Sci. Technol. 8, 128 (1997) 


\section{Figure captions}

Figure 1. Schematic diagram of the EP-SAW cyclone.

Figure 2. The SAW phase as a function of time showing a sequence of increasing collection plate bias voltages for a particulate size of $2.7 \mu \mathrm{m}$.

Figure 3. The data from figure 2 plotted as rate of phase change as a function of applied bias. 
Figure 1.

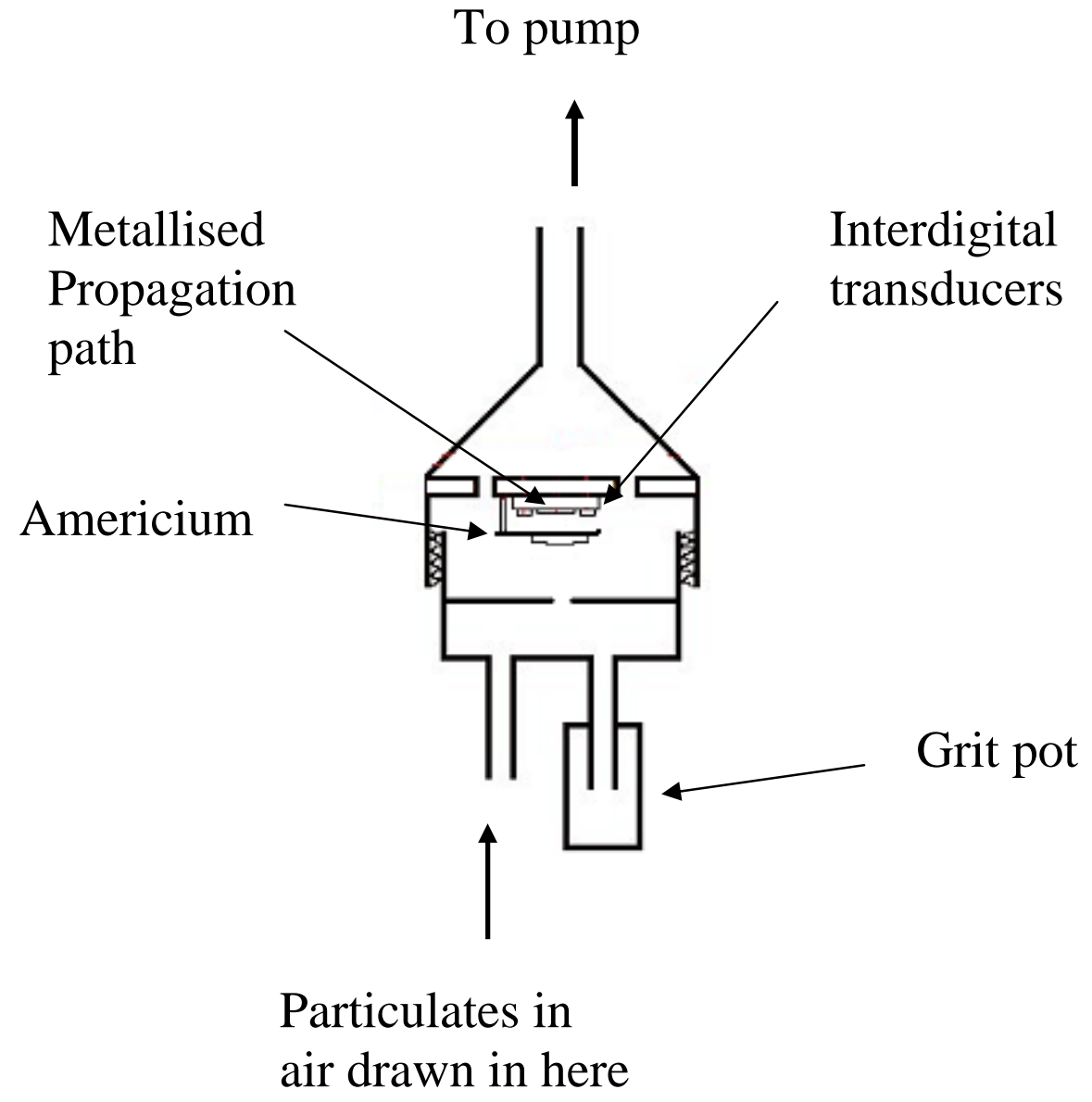


Figure 2.

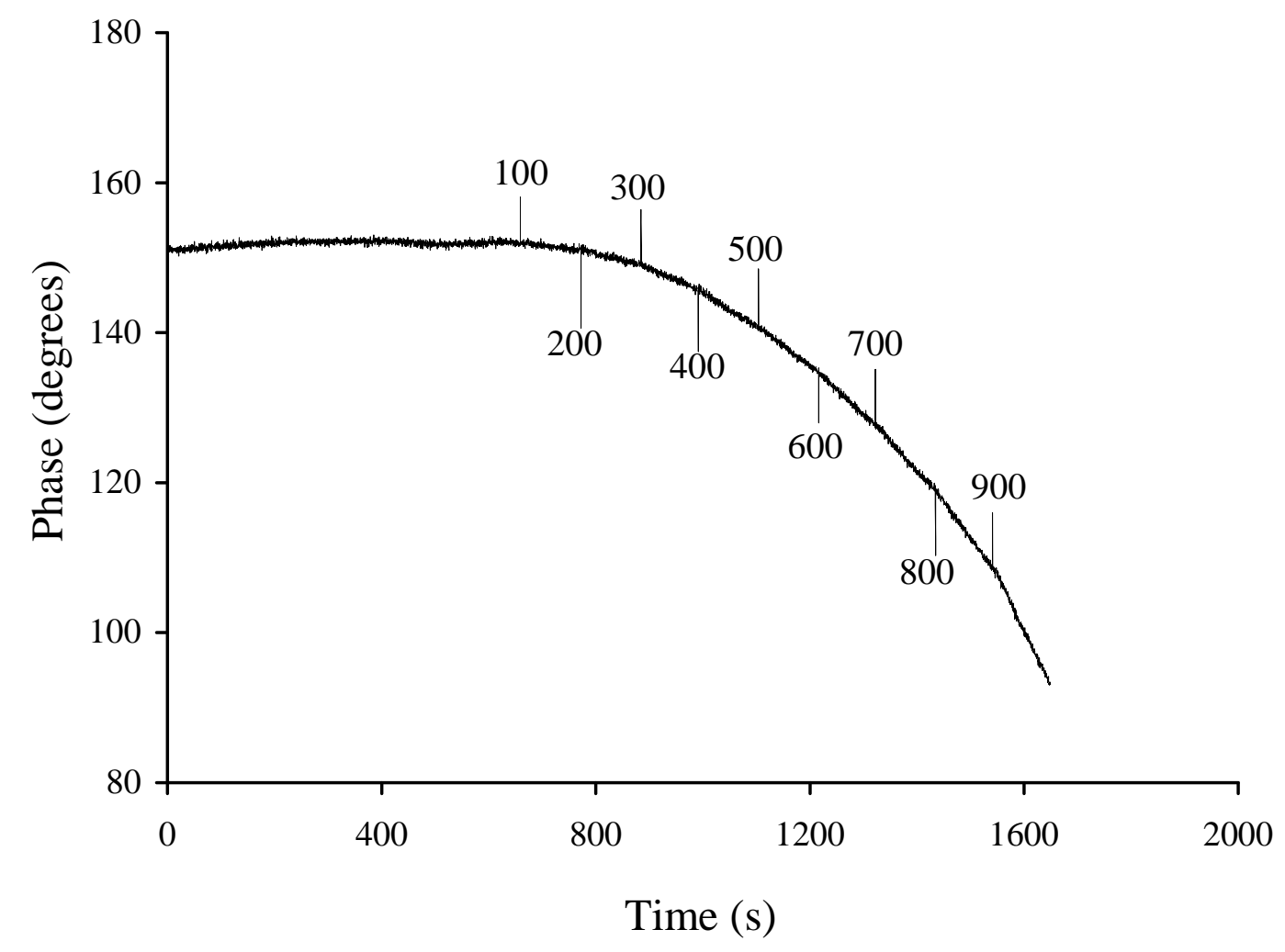


Figure 3.

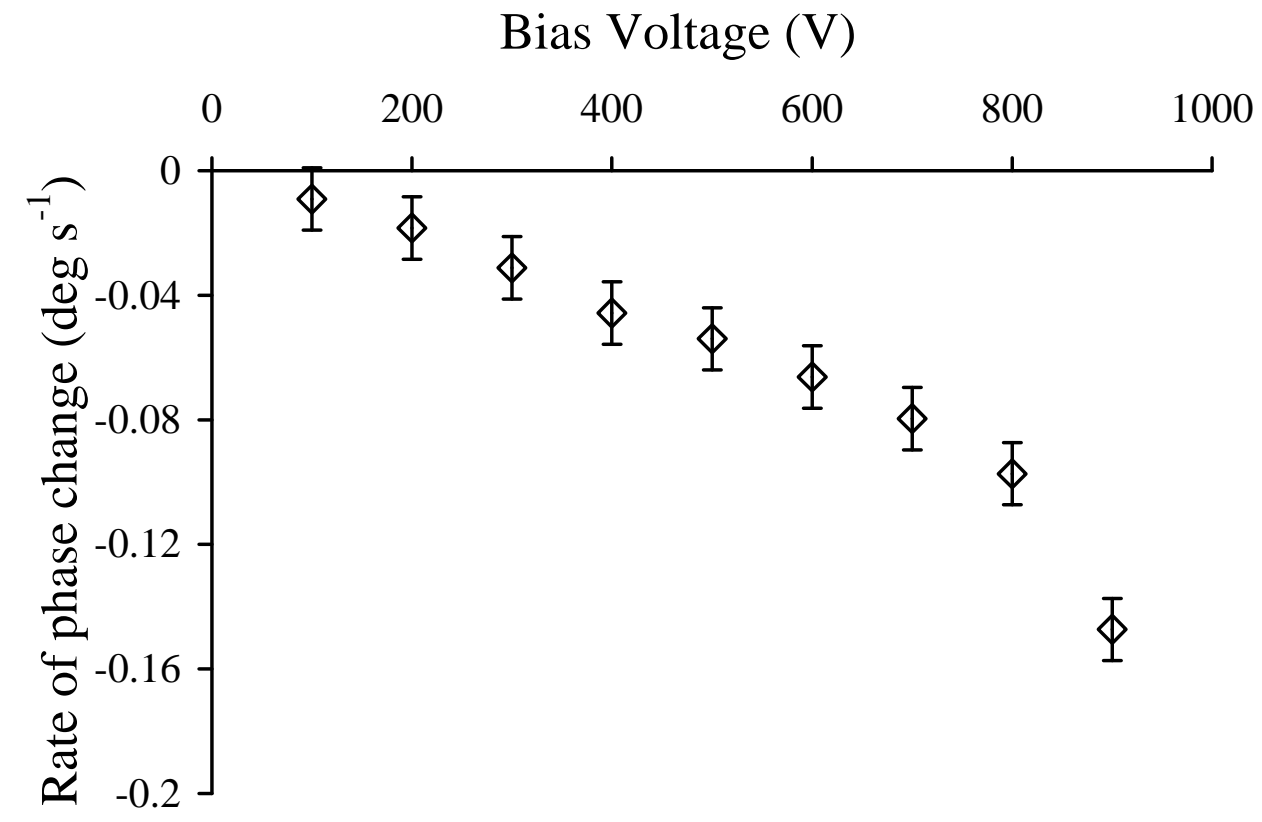

